\title{
The charge and mass perturbative renormalization in explicitly covariant LFD
}

\author{
J.-J. Dugne ${ }^{a *} \quad$ V.A. Karmanov ${ }^{b \dagger}$ \\ J.-F. Mathiot ${ }^{a \ddagger}$ \\ a) Laboratoire de Physique Corpusculaire, Université Blaise Pascal, \\ CNRS/IN2P3, 24 avenue des Landais, F-63177 Aubière Cedex, France \\ $\left.{ }^{b}\right)$ Lebedev Physical Institute, Leninsky Prospekt 53, 117924 Moscow, Russia
}

\begin{abstract}
In several preceding studies, the explicitly covariant formulation of light front dynamics was developed and applied to many observables. In the present study we show how in this approach the renormalization procedure for the first radiative correction can be carried out in a standard way, after separating out the contributions depending on the orientation of the light-front plane. We calculate the renormalized QED vertices $\gamma e^{-} \rightarrow e^{-}$and $e^{+} e^{-} \rightarrow \gamma$ and the electron self-energy and recover, in a straightforward way, the well known analytical results obtained in the ¿Feynman approach.
\end{abstract}

PACS numbers: 11.10.Gh, 11.25.Db, 11.30.Cp

Keywords: Renormalization, Light Front Dynamics

PCCF RI 00-13

*e-mail: dugne@clermont.in2p3.fr

$\dagger$ e-mail: karmanov@sci.lebedev.ru

‡e-mail: mathiot@in2p3.fr 


\section{Introduction}

Light Front Dynamics is a field theoretical approach which has been successfully applied to relativistic composite systems. The two forms of this scheme are: the standard Light Front Dynamics (LFD) [1] and explicitly Covariant Light Front Dynamics (CLFD) [2]. While the standard LFD deals with the state vector defined on the plane $t+z=0$, this plane is defined in CLFD by the invariant equation $\omega \cdot x=0$, where $\omega$ is a four-vector with $\omega^{2}=0$. The particular choice of the four-vector $\omega=(1,0,0,-1)$ turns CLFD into standard LFD.

In this article, we apply CLFD to the calculation, in first order perturbation theory, of the QED $\gamma e^{-} \rightarrow e^{-}$and $e^{+} e^{-} \rightarrow \gamma$ vertices and of the fermion self-energy. We will illustrate in details the calculational techniques of CLFD in order to point out its differences and similarities with respect to standard LFD and to the Feynman techniques.

The main difference with calculations in the Feynman approach lies in the fact that in CLFD, like in ordinary LFD, all the four-momenta, even in the intermediate states, are on their mass shells, whereas the amplitudes may be off-energy shells. Moreover, the amplitude may depend, in a well defined manner, on the orientation of the lightfront plane, i.e., on the four-vector $\omega$. This dependence is an unphysical one for physical amplitudes. Thus explicit covariance allows to disentangle clearly the physical amplitudes from unphysical ones. In this respect, the CLFD calculations differ from the standard LFD ones.

The study of the perturbative renormalization has already been done in standard LFD [3]- 9], resulting in the non-locality of the necessary counterterms. In our covariant approach this nonlocality manifests itself only in the terms depending on the orientation of the light front plane. These terms can be explicitly removed from the physical amplitude. We will show that after their separation, the renormalization of the $\omega$-independent part of the amplitude is carried out in a very simple way, like in the Feynman approach, and does not require any non-local counterterms. Then we find that the on-energy shell electromagnetic vertex in CLFD coincides with the on-mass shell Feynman vertex. The same is true for the electron self-energy.

In sect.2, we start with the calculation of the electron electromagnetic vertex in CLFD: the anomalous magnetic moment of the electron, and the renormalized electron charge. In sect.3 we apply our formalism to the vertex $e^{+} e^{-} \rightarrow \gamma$ for the threshold value of the photon momentum $Q^{2}=4 m^{2}$, and discuss the physical infrared singularity. The renormalized electron mass operator is calculated in sect. 4 . Sect.5 contains our concluding remarks. Some technical details are given in the appendices $\mathrm{A}$ and $\mathrm{B}$.

\section{The electron electromagnetic vertex}

\subsection{The anomalous magnetic moment of the electron}

The anomalous magnetic moment of the electron is a simple example of a higher order process in QED. Its calculation gives a finite result and does not require renormalization. It allows us also to show how to disentangle $\omega$-dependent terms in our formalism.

The spin 1/2 electromagnetic vertex in CLFD has the general form:

$$
J_{\rho}(q)=\bar{u}\left(p^{\prime}\right) \Gamma_{\rho} u(p),
$$


where $q=p^{\prime}-p$. We shall denote $Q^{2}=-q^{2}$. Due to the explicit covariance of our approach, the vertex operator $\Gamma_{\rho}$, according to ref. [10], can be decomposed into:

$$
\Gamma_{\rho}=F_{1} \gamma_{\rho}+\frac{i F_{2}}{2 m} \sigma_{\rho \nu} q^{\nu}+B_{1}\left(\frac{\hat{\omega}}{\omega \cdot p}-\frac{1}{(1+\eta) m}\right) P_{\rho}+B_{2} \frac{m}{\omega \cdot p} \omega_{\rho}+B_{3} \frac{m^{2}}{(\omega \cdot p)^{2}} \hat{\omega} \omega_{\rho},
$$

where $\sigma^{\rho \nu}=i\left(\gamma^{\rho} \gamma^{\nu}-\gamma^{\nu} \gamma^{\rho}\right) / 2, \hat{\omega}=\omega_{\mu} \gamma^{\mu}, \eta=Q^{2} /\left(4 m^{2}\right)$ and $m$ is the electron mass. The electromagnetic vertex (11) is gauge invariant since $J_{\rho} q^{\rho}=0$ (with the condition $\omega \cdot q=0$ ). The possible non-gauge-invariant terms are forbidden by $T$-invariance. The anomalous magnetic moment is the value of $F_{2}\left(Q^{2}\right)$ for $Q^{2}=0$.

The physical form factors $F_{1}$ and $F_{2}$ can easily be extracted from the vertex function $\Gamma_{\rho}$. To this end, we multiply $J_{\rho}$ by $\left[\bar{u}^{\sigma^{\prime}}\left(p^{\prime}\right) \gamma^{\rho} u^{\sigma}(p)\right]^{*},\left[\bar{u}^{\sigma^{\prime}}\left(p^{\prime}\right) i \sigma^{\rho \nu} q_{\nu} /(2 m) u^{\sigma}(p)\right]^{*}$, etc. and sum over polarizations. After taking the trace, we obtain the following quantities:

$$
\begin{aligned}
& c_{1}=\operatorname{Tr}\left[O_{\rho} \gamma^{\rho}\right], \quad c_{2}=\operatorname{Tr}\left[O_{\rho} i \sigma^{\rho \nu} q_{\nu}\right] /(2 m), \quad c_{3}=\operatorname{Tr}\left[O_{\rho}(\hat{\omega} / \omega \cdot p-1 /(1+\eta) m)\right] P^{\rho}, \\
& c_{4}=\operatorname{Tr}\left[O_{\rho}\right] \omega^{\rho} m / \omega \cdot p, \quad c_{5}=\operatorname{Tr}\left[O_{\rho} \hat{\omega}\right] \omega^{\rho} m^{2} /(\omega \cdot p)^{2},
\end{aligned}
$$

where

$$
O_{\rho}=\left(\hat{p}^{\prime}+m\right) \Gamma_{\rho}(\hat{p}+m) /\left(4 m^{2}\right) .
$$

With the decomposition (2) of $\Gamma_{\rho}$, we get a linear system of five equations for $F_{1}, F_{2}, B_{1-3}$ with the inhomogeneous part determined by $c_{1-5}$. Solving this system relative to $F_{2}$, we find:

$$
F_{2}=\frac{1}{4 \eta(1+\eta)^{2}}\left[\left(c_{3}+4 c_{4}-2 c_{1}\right)(1+\eta)+2\left(c_{1}+c_{2}\right)-2\left(c_{5}+c_{4}\right)(1+\eta)^{2}\right] .
$$

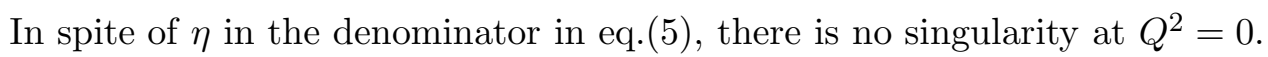

In the usual formulation of LFD on the plane $t+z=0$, the form factors of spin $1 / 2$ systems are found from the plus-component of the current, i.e., in our notation, from the contraction of $J_{\rho}$ in eq.(1), with $\omega_{\rho}$. This contraction gets rid of the contributions of $B_{2,3}$, but not the term proportional to $B_{1}$. The form factors $F_{1}^{\prime}$ and $F_{2}^{\prime}$ inferred in this way are thus given by:

$$
\begin{aligned}
J \cdot \omega & =\bar{u}^{\prime}\left[F_{1} \hat{\omega}+\frac{i F_{2}}{2 m} \sigma_{\rho \nu} \omega^{\rho} q^{\nu}+2 B_{1}\left(\hat{\omega}-\frac{\omega \cdot p}{(1+\eta) m}\right)\right] u \\
& \equiv \bar{u}^{\prime}\left[F_{1}^{\prime} \gamma_{\rho}+\frac{i F_{2}^{\prime}}{2 m} \sigma_{\rho \nu} q^{\nu}\right] u \omega^{\rho}
\end{aligned}
$$

where

$$
F_{1}^{\prime}=F_{1}+\frac{2 \eta B_{1}}{1+\eta}, \quad F_{2}^{\prime}=F_{2}+\frac{2}{1+\eta} B_{1} .
$$

The $B_{1}$ expression can be found from the above mentioned system of equations, leading to:

$$
B_{1}=-\frac{1}{8 \eta(1+\eta)}\left[\left(c_{3}+4 c_{4}-2 c_{1}\right)(1+\eta)+2\left(c_{1}+c_{2}\right)-4 c_{5}(1+\eta)^{2}\right] .
$$

Substituting $F_{2}$ from (5) and $B_{1}$ from (8) into eq. (7) for $F_{2}^{\prime}$, we get:

$$
F_{2}^{\prime}=\left(c_{5}-c_{4}\right) /(2 \eta) \text {. }
$$

Of course, in a given order of perturbation theory, both methods for calculating the form factors, by eqs.(5) and (9), should give the same result. This means we should find $B_{1}=0$. We shall see below that it is indeed the case. 


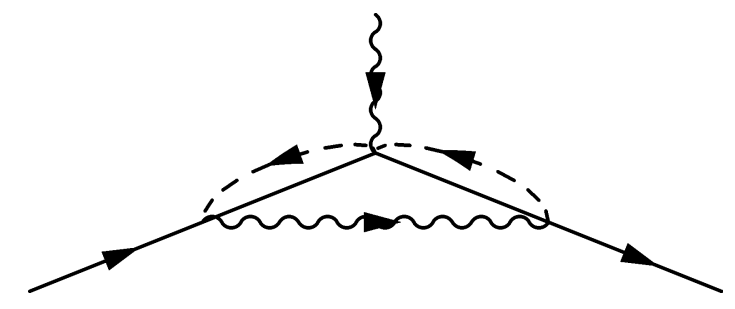

Figure 1: Electromagnetic vertex of the electron. The dashed line represents the spurion line, as explained in details in ref.[2]

Let us first calculate the form factor $F_{2}^{\prime}$, for $Q^{2}=0$. The amplitude corresponding to the graph of fig. 11 is given by the rules of the graph techniques [2] and has the form:

$$
\begin{aligned}
\bar{u}(p) \Gamma^{\rho} u(p) & =e^{2} \int \bar{u}(p) \gamma_{\mu}(\hat{p}-\hat{k}+m) \theta(\omega \cdot(p-k)) \delta\left(\left(p+\omega \tau_{1}-k\right)^{2}-m^{2}\right) \frac{d \tau_{1}}{\tau_{1}-i 0} \\
& \times \gamma^{\rho}(\hat{p}-\hat{k}+m) \theta(\omega \cdot(p-k)) \delta\left(\left(p+\omega \tau_{2}-k\right)^{2}-m^{2}\right) \frac{d \tau_{2}}{\tau_{2}-i 0} \gamma_{\nu} u(p) \\
& \times\left(-g^{\mu \nu}\right) \theta(\omega \cdot k) \delta\left(k^{2}-\mu^{2}\right) \frac{d^{4} k}{(2 \pi)^{3}} .
\end{aligned}
$$

We use the Feynman gauge for the photon propagator. The factor $\hat{p}-\hat{k}=\hat{k}_{1}-\hat{\omega} \tau_{1}=$ $\hat{k}_{2}-\hat{\omega} \tau_{2}$ includes the contact terms $-\hat{\omega} \tau_{1}$ and $-\hat{\omega} \tau_{2}$, as explained in ref. [2]. For the regularization of subsequent calculations, we introduced in (10) the photon mass $\mu$, although it is not necessary in the present subsection.

Integrating over $\tau_{1}, \tau_{2}$ and $k_{0}$, we get:

$$
\bar{u}(p) \Gamma^{\rho} u(p)=e^{2} \int \frac{\bar{u}(p) G^{\rho} u(p)}{\left(s-m^{2}\right)^{2}(1-x)^{2}} \frac{d^{3} k}{2 \varepsilon_{k}(2 \pi)^{3}},
$$

where $s=\left(k+k_{1}\right)^{2}=\left(k+k_{2}\right)^{2}, x=\omega \cdot k / \omega \cdot p$ and we note:

$$
G^{\rho}=-\gamma_{\mu}(\hat{p}-\hat{k}+m) \gamma^{\rho}(\hat{p}-\hat{k}+m) \gamma^{\mu} .
$$

The integrands for the scalar functions $c_{1-5}$ are represented in terms of the scalar products between the four-momenta $p, k$ and $\omega$. The scalar product $p \cdot k$ is given by:

$$
p \cdot k=\mu^{2} / 2+(1-x)\left(s-m^{2}\right) / 2,
$$

whereas the scalar products $\omega \cdot k$ and $\omega \cdot p$ always appear in the ratio $x$, with $0 \leq x \leq 1$.

It is convenient to introduce the variable $R=k-x p$. As usual (see, e.g., ref.[2]), we represent the spatial part of $R$ as $\vec{R}=\vec{R}_{\|}+\overrightarrow{R_{\perp}}$, where $\vec{R}_{\|}$is parallel to $\vec{\omega}$ and $\vec{R}_{\perp}$ is orthogonal to $\vec{\omega}$. Since, by definition of $R, R \cdot \omega=R_{0} \omega_{0}-\vec{R}_{\|} \cdot \vec{\omega}=0$, it follows that $R_{0}=\left|\vec{R}_{\|}\right|$, and, hence, $\vec{R}_{\perp}^{2}=-R^{2}$ is invariant. In terms of $\vec{R}_{\perp}^{2}$ and $x$, the variable $s$ writes:

$$
s=\frac{\vec{R}_{\perp}^{2}+\mu^{2}}{x}+\frac{\vec{R}_{\perp}^{2}+m^{2}}{1-x} .
$$


and the integration volume is transformed as: $d^{3} k / \varepsilon_{k}=d^{2} R_{\perp} d x / x$.

Substituting these expressions into (11), we find (for $\mu=0$ ):

$$
\bar{u}(p) \Gamma^{\rho} u(p)=\frac{\alpha}{4 \pi^{2}} \int \bar{u}(p) G^{\rho} u(p) \frac{x d x d^{2} R_{\perp}}{\left(\vec{R}_{\perp}^{2}+x m^{2}\right)^{2}},
$$

where we denote $\alpha=e^{2} / 4 \pi$. To calculate $F_{2}^{\prime}$ by eq.(9) (for $\eta \rightarrow 0$ ), we substitute $\Gamma_{\rho}$ from (14) into (雨) (for $p^{\prime}=p$ ) and then into expressions (3) for $c_{4}$ and $c_{5}$. Calculating the traces, we get:

$$
F_{2}^{\prime}(0)=\frac{\alpha}{4 \pi^{2}} \int 4 m^{2} x(1-x) \frac{x d x d^{2} R_{\perp}}{\left(\vec{R}_{\perp}^{2}+x m^{2}\right)^{2}} .
$$

We thus obtain the well known result for the anomalous magnetic moment of the electron:

$$
F_{2}^{\prime}(0)=\frac{\alpha}{2 \pi} .
$$

Now consider the form factor $F_{2}$ calculated after separation of the $\omega$-dependent terms. According to (7), it is related to $F_{2}^{\prime}$ by $F_{2}(0)=F_{2}^{\prime}(0)-2 B_{1}(0)$. From eq. (8), for $Q^{2}=0$, we find the following expression for $B_{1}$ :

$$
B_{1}(0)=\frac{\alpha}{2 \pi} \int \frac{\left[m^{2}(2-x) x^{2}-2 R_{\perp}^{2}(1-x)-\mu^{2}(2-x)\right]}{\left[R_{\perp}^{2}+m^{2} x^{2}+\mu^{2}(1-x)\right]^{2}} R_{\perp} d R_{\perp} d x
$$

which is logarithmically divergent. We regularize it using the Pauli-Villars method, i.e., the photon propagator is replaced by:

$$
\frac{1}{k^{2}-\mu^{2}} \rightarrow \frac{1}{k^{2}-\mu^{2}}-\frac{1}{k^{2}-\Lambda^{2}}
$$

In the absence of infrared singularity we can put in (18) $\mu=0$. Hence, the regularized expression for $B_{1}$ reads:

$$
B_{1}^{r e g}=B_{1}(\mu=0)-B_{1}(\mu=\Lambda)
$$

Integrating it over $R_{\perp}$, we get:

$$
B_{1}^{r e g}=-\frac{\alpha}{4 \pi} \int_{0}^{1} d x \frac{d}{d x}\left[x(2-x) \log \left(\frac{\Lambda^{2}(1-x)+m^{2} x^{2}}{m^{2} x^{2}}\right)\right] .
$$

After integration over $x$ we get $B_{1}^{r e g}=0$ for any value of $\Lambda$. This clearly shows that both methods to calculate the anomalous magnetic moment of the electron give the same result.

\subsection{The renormalized electron charge}

In order to calculate the radiative correction to the form factor $F_{1}$, we have to renormalize the charge. The renormalization means that the Lagrangian contains a counter term of the form:

$$
Z_{1} \bar{\psi} \gamma^{\rho} \psi A_{\rho}
$$

hence, the amplitude $J^{\rho}$ is replaced by

$$
J^{\rho} \rightarrow J_{r e n}^{\rho}=J^{\rho}-J_{0}^{\rho},
$$


where

$$
J_{0}^{\rho}=Z_{1} \bar{u}\left(p^{\prime}\right) \gamma^{\rho} u(p) .
$$

The renormalization procedure is described in many textbooks, see for example [11, 12, 13]. In order to find $Z_{1}$, one must calculate the amplitude $\bar{u}(p) \Gamma^{\rho} u(p)$ from the diagram of fig. 1. The value of $Z_{1}$ is in fact just the form factor $F_{1}(0)$ determined by this diagram. For $p=p^{\prime}$ the general decomposition (1) turns into

$$
\bar{u}(p) \Gamma^{\rho} u(p)=Z_{1} \bar{u}(p) \gamma^{\rho} u(p)+Z^{\prime} \frac{\omega^{\rho} m}{\omega \cdot p} \bar{u}(p) u(p),
$$

where $Z_{1}=F_{1}(0)$ and $Z^{\prime}=B_{2}(0)+B_{3}(0)$.

From (21) the constant $Z_{1}$ is given by:

$$
Z_{1}=\frac{1}{4 \omega \cdot p} \operatorname{Tr}\left[\omega_{\rho} \Gamma^{\rho}(\hat{p}+m)\right] .
$$

The vertex $\Gamma^{\rho}$ is determined by eq.(10) and is reduced to (11). For regularization purposes, we should now keep the photon mass $\mu$ finite. From eq.(22) we find:

$$
Z_{1}=\frac{\alpha}{(2 \pi)^{3}} \int d^{2} R_{\perp} \int_{0}^{1} \frac{\left[\vec{R}_{\perp}^{2}+m^{2}\left(-2+2 x+x^{2}\right)\right] x}{\left[\vec{R}_{\perp}^{2}+m^{2} x^{2}+\mu^{2}(1-x)\right]^{2}} d x
$$

The subsequent calculation is similar to the calculation given in appendix A. That is, we calculate $Z_{1}(\mu, L)$ for a fixed upper limit $L$ of the variable $R_{\perp}$ in the integral (23), take the difference $Z_{1}(\mu, L)-Z_{1}(\Lambda, L)$, take the limit $L \rightarrow \infty$ and then calculate the limits $\mu \rightarrow 0$ and $\Lambda \rightarrow \infty$. We then obtain:

$$
Z_{1}(\mu \rightarrow 0, \Lambda \rightarrow \infty)=\frac{9 \alpha}{8 \pi}+\frac{\alpha}{2 \pi} \log \left(\frac{\mu^{2}}{m^{2}}\right)+\frac{\alpha}{4 \pi} \log \left(\frac{\Lambda^{2}}{m^{2}}\right) .
$$

This expression exactly coincides with the expression found in the Feynman formalism 14. We emphasize that this result for $Z_{1}$ is obtained for the physical part of the full vertex (21), after separating out the unphysical term proportional to $Z^{\prime} \omega_{\rho}$. The latter term can be disregarded, there is no need to calculate it.

\section{Application to the vertex $e^{+} e^{-} \rightarrow \gamma$}

As a direct application of the preceding calculation, let us now consider the leptonic decay width of the positronium. In the Weisskopf-Van Royen limit, the decay width is proportional to the elementary vertex $e^{+} e^{-} \rightarrow \gamma$, where the $e^{+} e^{-}$pair originates from the positronium wave function with zero relative momentum, i.e. with $p_{e^{+}}=p_{e^{-}}=p$.

\subsection{On-energy-shell spin structure}

The on-shell amplitude for the process $e^{+} e^{-} \rightarrow \gamma$ depends on the four-vectors $p$ and $\omega$. Its general structure thus reads:

$$
\bar{u}(p) M^{\rho} v(p)=A \bar{u}(p) \gamma^{\rho} v(p)+B \frac{p^{\rho}}{\omega \cdot p} \bar{u}(p) \hat{\omega} v(p)+C \frac{\omega^{\rho} m^{2}}{(\omega \cdot p)^{2}} \bar{u}(p) \hat{\omega} v(p) .
$$


Here $v(p)$ is the positron spinor. The constant $A$ in (25) is the value of the form factor $F_{1}\left(Q^{2}\right)$ at $Q^{2}=4 m^{2}$. One can also construct the structure $\sigma^{\rho \beta} \omega_{\beta} / \omega \cdot p$, but it is not independent, since:

$$
\frac{i m}{\omega \cdot p} \bar{u}(p) \sigma^{\rho \beta} \omega_{\beta} v(p)=\bar{u}(p) \gamma^{\rho} v(p)-\frac{p^{\rho}}{\omega \cdot p} \bar{u}(p) \hat{\omega} v(p) .
$$

Multiplying (25) on the left by $u(p)$ and on the right by $\bar{v}(p)$ and summarizing over polarizations, we get the factors $\sum_{\lambda} u(p) \bar{u}(p)=(\hat{p}+m), \sum_{\lambda} v(p) \bar{v}(p)=(\hat{p}-m)$. We introduce therefore the quantity:

$$
\tilde{M}^{\rho}=(\hat{p}+m) M^{\rho}(\hat{p}-m)
$$

and calculate the following traces:

$$
\begin{aligned}
& T_{1} \equiv \frac{1}{16 m^{2}} \operatorname{Tr}\left[\tilde{M}^{\rho} \gamma_{\rho}\right]=(-3 A+C) / 2, \\
& T_{2} \equiv \frac{1}{16 m^{2}} \operatorname{Tr}\left[\tilde{M}^{\rho} \hat{\omega}\right] \frac{p_{\rho}}{\omega \cdot p}=(B+C) / 2, \\
& T_{3} \equiv \frac{1}{16 m^{2}} \operatorname{Tr}\left[\tilde{M}^{\rho} \hat{\omega}\right] \frac{\omega_{\rho} m^{2}}{(\omega \cdot p)^{2}}=(A+B) / 2 .
\end{aligned}
$$

So we can find out the coefficients which determine the amplitude (25):

$$
\begin{aligned}
& A=T_{2}-T_{1}-T_{3} \\
& B=T_{1}-T_{2}+3 T_{3} \\
& C=-T_{1}+3 T_{2}-3 T_{3} .
\end{aligned}
$$

\subsection{The physical amplitude}

The off-energy-shell amplitude depicted in fig.2 is given by the rules of the graph techniques [2] and writes:

$$
\begin{aligned}
\bar{u}(p) M_{1}^{\rho} v\left(p^{\prime}\right) & =e^{2} \int \bar{u}(p) \gamma_{\mu}(\hat{k}+m) \theta(\omega \cdot k) \delta\left(k^{2}-m^{2}\right) \frac{d^{4} k}{(2 \pi)^{3}} \\
& \times \gamma^{\rho}(m-(\hat{Q}-\hat{k})) \theta(\omega \cdot(Q-k)) \delta\left(\left(Q-k+\omega \tau_{2}\right)^{2}-m^{2}\right) \frac{d \tau_{2}}{\tau_{2}-i 0} \\
& \times \gamma_{\nu} v\left(p^{\prime}\right)\left(-g^{\mu \nu}\right) \delta\left(\left(p-\omega \tau^{\prime}+\omega \tau_{1}-k\right)^{2}-\mu^{2}\right) \theta(\omega \cdot(p-k)) \frac{d \tau_{1}}{\tau_{1}-i 0} .
\end{aligned}
$$

where $Q=q-\omega \tau$. Note that the fermion and antifermion propagators in LFD differ from each other. The propagator $(\hat{k}+m)$ in (29) corresponds to the electron, whereas the propagator $(m-(\hat{Q}-\hat{k}))$ corresponds to the positron.

The factor $m-(\hat{Q}-\hat{k})=m-\left(\hat{k}_{1}-\hat{\omega} \tau_{2}\right)$ incorporates the difference $\hat{k}_{1}-\hat{\omega} \tau_{2}$ and, therefore, takes into account the contact term $-\hat{\omega} \tau_{2}$. We consider in this section the case $Q^{2}=4 m^{2}$ relevant for the decay width of the positronium, so that $p^{\prime}=p, \tau^{\prime}=0$ and so the two graphes corresponding to the two different time orderings should give the same contribution $M^{\rho}=M_{1}^{\rho}+M_{2}^{\rho}=2 M_{1}^{\rho}$. 


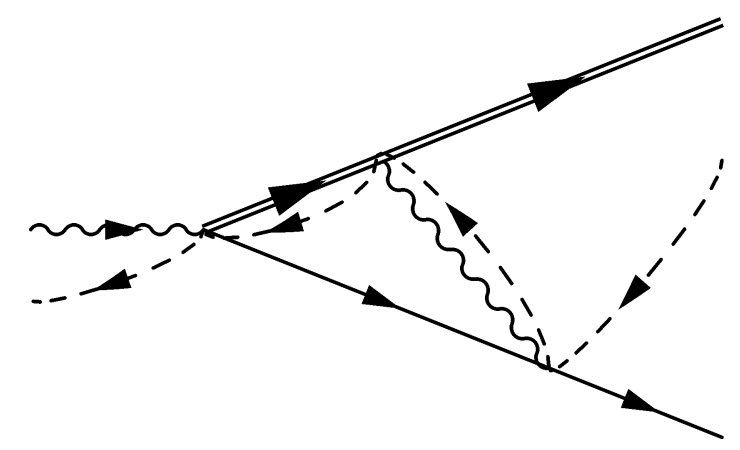

Figure 2: Light-front time ordered graph for $e^{+} e^{-} \rightarrow \gamma$. A similar diagram with the opposite time-ordering for the photon exchange should be added.

After integration over $\tau_{1}$ and $\tau_{2}$, the amplitude $(29)$ is given by:

$$
\begin{aligned}
\bar{u}(p) M_{1}^{\rho} v(p) & =-e^{2} \int \bar{u}(p) \gamma_{\mu}(\hat{k}+m) \gamma^{\rho}(m-(\hat{Q}-\hat{k})) \gamma^{\mu} v(p) \\
& \times \frac{\theta(\omega \cdot(p-k)) \theta(\omega \cdot(Q-k)) \theta(\omega \cdot k)}{\left(s_{12}-Q^{2}\right)\left(1-\frac{\omega \cdot k}{\omega \cdot Q}\right)\left(s_{123}-Q^{2}\right)\left(\frac{\omega \cdot p-\omega \cdot k}{\omega \cdot Q}\right)} \frac{d^{3} k}{2 \varepsilon_{k}(2 \pi)^{3}},
\end{aligned}
$$

where

$$
s_{12}-Q^{2}=2(\omega \cdot Q) \tau_{2}, \quad s_{123}-Q^{2}=2(\omega \cdot Q) \tau_{1}
$$

and

$$
\begin{aligned}
s_{12} & =\left(k+k_{1}\right)^{2}=\frac{\vec{R}_{k \perp}^{2}+m^{2}}{x_{k}}+\frac{\vec{R}_{k \perp}^{2}+m^{2}}{1-x_{k}}, \\
s_{123} & =\left(k+k_{2}+p\right)^{2}=\frac{\vec{R}_{k \perp}^{2}+m^{2}}{x_{k}}+\frac{\vec{R}_{k_{1} \perp}^{2}+\mu^{2}}{x_{k_{1}}}+\frac{\vec{R}_{p^{\prime} \perp}^{2}+m^{2}}{x_{p^{\prime}}} \\
& =\frac{\vec{R}_{k \perp}^{2}+m^{2}}{x_{k}}+\frac{\vec{R}_{k \perp}^{2}+\mu^{2}}{1 / 2-x_{k}}+2 m^{2} .
\end{aligned}
$$

Like in the previous section, we define above the variables $R_{l}=l-x_{l} Q$ with $x_{l}=\omega \cdot l / \omega \cdot Q$, where $l$ is either $k, k_{1}$ or $p^{\prime}$. At the threshold $Q^{2}=4 m^{2}$, we have $\vec{R}_{p^{\prime}} \perp=0$ and $x_{p^{\prime}}=1 / 2$ in the variable $s_{123}$. We thus find:

$$
\tilde{M}^{\rho}=-2 \frac{e^{2}}{(2 \pi)^{3}} \int d^{2} R_{\perp} \int_{0}^{1 / 2} \frac{O^{\rho}}{\left(s_{12}-4 m^{2}\right)(1-x)\left(s_{123}-4 m^{2}\right)(1 / 2-x)} \frac{d x}{2 x},
$$

where:

$$
O^{\rho}=(\hat{p}+m) \gamma_{\mu}(\hat{k}+m) \gamma^{\rho}(m-(\hat{Q}-\hat{k})) \gamma^{\mu}(\hat{p}-m)
$$


The amplitude $\tilde{M}^{\rho}$ is connected to $M^{\rho}$ by eq.(26). The factor 2 in (32) results from the sum of the two amplitudes $M_{1}$ and $M_{2}$. In order to find the coefficients $A, B, C$ which determine the amplitude (25), we substitute $\tilde{M}^{\rho}$ into eqs.(27), (28), regularize the expression by the Pauli-Villars prescription:

$$
A \rightarrow A(\mu)-A(\Lambda)
$$

(and similarly for $B, C$ ), and take the limits $\mu \rightarrow 0, \Lambda \rightarrow \infty$. The details of the calculation are given in the appendix A. The final expression for $A$ is then:

$$
A=\frac{\alpha m}{\mu}-\frac{7 \alpha}{8 \pi}+\frac{\alpha}{2 \pi} \log \left(\frac{\mu^{2}}{m^{2}}\right)+\frac{\alpha}{4 \pi} \log \left(\frac{\Lambda^{2}}{m^{2}}\right) .
$$

This expression exactly coincides with that calculated in the Feynman formalism [14].

The integral for $B(\mu)$ converges and does not depend on $\mu$ :

$$
B(\mu)=-\frac{\alpha}{4 \pi} \text {. }
$$

The amplitude regularized à la Pauli-Villars is determined by the difference $B_{\text {reg }}=B(\mu)-$ $B(\Lambda)$. It is therefore zero.

From (34) and (24) we recover the renormalized amplitude

$$
A_{\text {ren }}=A-Z=\frac{\alpha m}{\mu}-\frac{2 \alpha}{\pi},
$$

which coincides with the result found in the Feynman approach [14]. It contains the term $\frac{\alpha m}{\mu}$, corresponding to an infrared singularity. In the next section we will show that this singularity is physical but does not contribute to the relativistic correction to the decay width of the positronium.

The calculation of $C$ gives a divergent result even after a single Pauli-Villars regularization. However, $C$ is the coefficient in front of the term $\omega^{\rho} \bar{u} \hat{\omega} u$ which is proportional to $\omega^{\rho}$. Like $Z^{\prime}$ in eq. (21), it is separated out in eq. (25) and does not contribute to the observable amplitude, determined by $A$.

\subsection{Infrared singularity}

The infrared singularity in (36) results from the Coulomb interaction between the electron and the positron. It manifests itself in the low momentum limit in the loop of fig.2.

To calculate this limit, it is sufficient to take the nonrelativistic limit for all the four momenta. It means that we make in the numerator of eq.(30) the following replacements:

$$
\bar{u}(p) \gamma_{\mu}(\hat{k}+m) \rightarrow \bar{u}(p)\left(1+\gamma_{0}\right) m \quad \text { and } \quad(m-(\hat{Q}-\hat{k})) \gamma^{\mu} v(p) \rightarrow-m\left(1-\gamma_{0}\right) v(p) .
$$

We used the fact that in this equation only the matrix $\gamma_{\mu}$ with $\mu=0$ contributes. After this substitution, the integral (30) converges and its calculation, in the $\mu \rightarrow 0$ limit, gives

$$
2 \bar{u}(p) M_{1}^{\rho} v(p)=\frac{\alpha m}{\mu} \bar{u}(p) \gamma^{\rho} v(p) .
$$

This Coulomb contribution has to be removed from the total radiative corrections (36) since it is already taken into account in the calculation of the positronium wave function. After this, we reproduce the well known radiative correction $-\frac{2 \alpha}{\pi}$.

This result enables us to calculate the relativistic radiative corrections beyond the Weisskopf-Van Royen approximation. This is done for instance in ref.[15] for the calculation of the leptonic decay width of the $J / \psi$. 


\section{The electron self-energy}

Since our formulation of LFD is explicitly covariant, we are able to follow very closely the standard procedure of renormalization of the fermion self-energy in perturbation theory. The self energy diagram is shown in Fig.3. As already done for the electromagnetic vertex, we can write down immediately the general spin structure of the self energy. It is very simple and given by:

$$
\Sigma(p)=A_{1}\left(p^{2}\right)+B_{1}\left(p^{2}\right) \frac{\hat{p}}{m}+C_{1}\left(p^{2}\right) \hat{\omega} .
$$

The coefficients $A_{1}, B_{1}, C_{1}$ are scalar functions of $p^{2}$ only. Here $p=p_{1}-\omega \tau_{1}$ is the total momentum entering the diagram, $p_{1}$ is the external fermion momentum, with $p_{1}^{2}=m^{2}$, and $\omega \tau_{1}$ is the external spurion momentum. The off-energy-shell mass operator $\Sigma(p)$ depends on the value $p^{2}=m^{2}-2(\omega \cdot p) \tau_{1}$.

Like in the above calculations, we subtract from $\Sigma(p)$ the $\omega$-dependent structure, introducing:

$$
\tilde{\Sigma}(p)=\Sigma(p)-C_{1}\left(p^{2}\right) \hat{\omega}=A_{1}\left(p^{2}\right)+B_{1}\left(p^{2}\right) \frac{\hat{p}}{m} .
$$

The standard procedure of renormalization of Feynman diagrams relies on two counterterms: the mass counterterm $\delta m^{2}$ and the wave function renormalization proportional to $Z_{2}$ [11. They can be incorporated explicitly in the Hamiltonian.

Following [13], the renormalized self energy $\Sigma_{R}(p)$ is defined as the part of $\tilde{\Sigma}(p)$ which is of second order in the variable $(\hat{p}-m)$. Without loss of generality, we can rewrite $\Sigma(p)$ in the form:

$$
\tilde{\Sigma}(p)=A_{0}+(\hat{p}-m) B_{0}+\Sigma_{R}(p) .
$$

Here $A_{0}, B_{0}$ are constants (they do not depend on $p^{2}$ ), and $\Sigma_{R}(p)$ is the renormalized self-energy written as:

$$
\Sigma_{R}(p)=(\hat{p}-m)^{2} \mathcal{M}(p),
$$

where the matrix $\mathcal{M}(p)$ can be represented as:

$$
\mathcal{M}(p)=a+(\hat{p}+m) b
$$

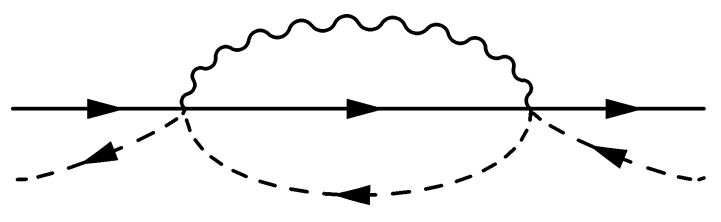

Figure 3: The electron self energy graph 
The renormalized fermion propagator now reads:

$$
\frac{1}{\hat{p}-m-(\hat{p}-m)^{2} \mathcal{M}(p)} \text {. }
$$

It has the same pole and the same residue at $\hat{p}=m$ as the free propagator of the physical fermion.

The explicit calculation of the renormalized fermion self energy is now straightforward. According to the rules of CLFD, the electron self energy shown on fig. 3 has the form:

$$
\begin{aligned}
-\Sigma(p)= & e^{2} \int \theta(\omega \cdot k) \delta\left(k^{2}-m^{2}\right) \gamma^{\mu}(\hat{k}-\hat{\omega} \tau+m) \gamma^{\nu}\left(-g_{\mu \nu}\right) \\
& \times \theta(\omega \cdot(p-k)) \delta\left((p+\omega \tau-k)^{2}-\mu^{2}\right) \frac{d \tau}{\tau-i 0} \frac{d^{4} k}{(2 \pi)^{3}} \\
= & -\frac{e^{2}}{(2 \pi)^{3}} \int \frac{4 m-2 \hat{k}+2 \hat{\omega} \tau}{s-p^{2}} \frac{d^{2} R_{\perp} d x}{2 x(1-x)} .
\end{aligned}
$$

As indicated in the previous section, we introduce the photon mass $\mu$ for infrared regularization. The term $\hat{\omega} \tau$ in (42) contributes to $C_{1}\left(p^{2}\right) \hat{\omega}$ only and can be omitted in the calculation of $\tilde{\Sigma}(p)$. In eq.(42), $\tau=\left(s-p^{2}\right) /(2 \omega \cdot p)$ and

$$
s=\frac{R_{\perp}^{2}+m^{2}}{x}+\frac{R_{\perp}^{2}+\mu^{2}}{1-x}, \quad k \cdot p=\frac{R_{\perp}^{2}+m^{2}}{x}-\frac{1}{2} x p^{2}
$$

with $x=\omega \cdot k / \omega \cdot p, R=k-x p$, and the phase-space volume is given by $d^{3} k / \varepsilon_{k}=$ $d^{2} R_{\perp} d x / x$.

Now, starting from eq. (42) we can calculate $\Sigma_{R}(p)$, and the scalar coefficients $a$ and $b$ in (41). Knowing $\Sigma(p)$ from eq.(42), we calculate the coefficients $A_{1}, B_{1}$ in (37) and find $\Sigma(p)$ by eq.(38) . Comparing (38) with (39), we express $A_{0}, B_{0}$ through $A_{1}, B_{1}$ for $p^{2}=m^{2}$. Using again the representation (39) for $\tilde{\Sigma}(p)$, we finally obtain the functions $a, b$, which determine the self-energy (40), through $A_{1}\left(p^{2}\right), B_{1}\left(p^{2}\right)$ and $A_{0}, B_{0}$. The details of the calculation are given in appendix $\mathrm{B}$. For the functions $a$ and $b$ we find:

$$
\begin{aligned}
a & =\frac{\alpha}{4 \pi m} \frac{1}{(1-\rho)}\left(1-\frac{2-3 \rho}{1-\rho} \log \rho\right) \\
b & =-\frac{\alpha}{2 \pi m^{2} \rho}\left[\frac{1}{2(1-\rho)}\left(2-\rho+\frac{\rho^{2}+4 \rho-4}{1-\rho} \log \rho\right)+1+\log \frac{\mu^{2}}{m^{2}}\right]
\end{aligned}
$$

where

$$
\rho=\frac{m^{2}-p^{2}}{m^{2}}
$$

With these expressions for $a$ and $b$ the renormalized mass operator writes:

$$
\Sigma_{R}(p)=(\hat{p}-m)^{2} \mathcal{M}(p)=(\hat{p}-m)^{2}[a+(\hat{p}+m) b] .
$$

It exactly coincides with the standard result given for instance in refs. [11, 13]. Note that in higher order calculations, the $\omega$-dependent term $C_{1}\left(p^{2}\right) \hat{\omega}$ may give an $\omega$ independent contribution, when $\Sigma(p)$ enters as a part of a more complex diagram. It can also be renormalized. The calculation of $C_{1}^{r e n}$ is given in appendix B. 


\subsection{The antifermion self-energy}

Since the forms of the fermion and antifermion propagators are different in LFD (they contain $(\hat{p}+m)$ for fermion and $-(\hat{p}-m)$ for antifermion), the form of the self-energy is also different. However, there is no need to repeat the calculation: the antifermion self-energy $\bar{\Sigma}(p)$ can be found from the fermion one.

We represent $\bar{\Sigma}(p)$ similarly to (37):

$$
\begin{aligned}
\bar{\Sigma}(p) & =\bar{A}_{1}\left(p^{2}\right)+\bar{B}_{1}\left(p^{2}\right) \frac{\hat{p}}{m}+\bar{C}_{1}\left(p^{2}\right) \hat{\omega} \\
& =\bar{A}_{0}+\bar{B}_{0}(\hat{p}+m)+\bar{C}_{1}\left(p^{2}\right) \hat{\omega}+\bar{\Sigma}_{R}(p),
\end{aligned}
$$

where $\bar{A}_{0}, \bar{B}_{0}$ are constants. The renormalized antifermion self-energy $\bar{\Sigma}_{R}(p)$ is represented in the form similar to (44):

$$
\bar{\Sigma}_{R}(p)=(\hat{p}+m)^{2}[\bar{a}-(\hat{p}-m) \bar{b}]
$$

One can easily see that

$$
\bar{a}=a \quad \bar{b}=b
$$

where $a, b$ are given by eq.(43). Indeed, from the comparison of the fermion and antifermion propagators we see that the antifermion self energy $\bar{\Sigma}_{R}(p)$ can be obtained from the fermion one $\Sigma_{R}(p)$ by:

$$
\bar{\Sigma}_{R}(p, m)=-\Sigma_{R}(p,-m)=-(\hat{p}+m)^{2}[a(-m)+(\hat{p}-m) b(-m)] .
$$

Comparing (46) with (48) and taking into account that $a(-m)=-a(m), b(-m)=b(m)$ (see the explicit expressions (43)) we reproduce eqs. (46) and (47).

\section{Conclusion}

The understanding of perturbative renormalization in QED is an unavoidable step before studying more subtle systems like QCD. While this perturbative renormalization is now a text-book section for the standard formulation of field theory using Feynman graph techniques, it is not as well understood in Light-Front Quantization. The main reason being the difficulty to exhibit the covariant structure of the electromagnetic vertex and electron self-energy since standard LFD breaks explicitly covariance.

We have shown in this study that the covariant formulation of LFD is a powerful tool in order to make the link between LFD and Feynman approaches. The explicit covariance of our formulation enables us to exhibit the relativistic structure of the electromagnetic vertex in QED, as well as the electron self-energy. We are thus in a position to extract, after renormalization, the finite physical contribution from the infinite amplitude. To do that, we have to know the dependence of the operators on the orientation, $\omega$, of the light front. This is trivial in CLFD. In the standard formulation of LFD, this dependence can not always be disentangled from the physical part of the amplitude. This $\omega$-dependent contribution is responsible for the non-locality of the counter terms needed to renormalize the infinite amplitude in LFD [3]- 9].

The finite physical amplitude we found in our approach for the electromagnetic vertex, the electron self-energy, and the $e^{+} e^{-} \rightarrow \gamma$ amplitude agree thus with the standard textbook results. 
We emphasize that in order to reproduce these results, a covariant regularization of divergences (Pauli-Villars in the present study) is important. The attempt to regularize the integrals by a cut-off in the variable $R_{\perp}$, for instance, allows of course to work with finite integrals. It gives finite, but however wrong, renormalized results.

The QED vertex $e^{+} e^{-} \rightarrow \gamma$, corrected by a color factor, coincides with the QCD vertex $q^{+} q^{-} \rightarrow \gamma$. This result is applied in [15] for the calculation of the relativistic radiative correction to the $J / \psi$ leptonic decay width.

The question of non-perturbative renormalization, for scalar particles, will be adressed in a forthcoming publication 16.

\section{Acknowledgements}

One of the author (V.A.K.) is sincerely grateful for the warm hospitality of the Laboratoire de Physique Corpusculaire, Université Blaise Pascal in Clermont-Ferrand, where this work was performed. This work was partially supported by the grant No. 99-02-17263 of the Russian Fund for Basic Researches.

\section{A Calculation of $A, B$ and $C$}

As explained in sect.3.2, we substitute $\tilde{M}^{\rho}$ into eqs.(27), (28) in order to find the coefficients $A, B, C$ determining the amplitude (25). We thus find:

$$
(A, B, C)=-\frac{8 \pi \alpha}{(2 \pi)^{3}} \int d^{2} R_{\perp} \int_{0}^{1 / 2} \frac{(a, b, c)}{\left(s_{12}-4 m^{2}\right)(1-x)\left(s_{123}-4 m^{2}\right)(1 / 2-x)} \frac{d x}{2 x},
$$

with:

$$
\begin{aligned}
a & =t_{2}-t_{1}-t_{3}=\frac{1}{16 m^{2}}\left\{\operatorname{Tr}\left[O^{\rho} \hat{\omega}\right] \frac{p_{\rho}}{\omega \cdot p}-\operatorname{Tr}\left[O^{\rho} \gamma_{\rho}\right]-\operatorname{Tr}\left[\tilde{M}^{\rho} \hat{\omega}\right] \frac{\omega_{\rho} m^{2}}{(\omega \cdot p)^{2}}\right\} \\
& =-\frac{1}{x}\left[\vec{R}_{\perp}^{2}(1-2 x)+m^{2}\left(1+4 x^{2}\right)\right], \\
b & =t_{1}-t_{2}+3 t_{3}=\frac{1}{x}\left[\vec{R}_{\perp}^{2}(1-4 x)+m^{2}(1-2 x)^{2}(1+2 x)\right], \\
c & =-t_{1}+3 t_{2}-3 t_{3}=-\frac{1}{4 m^{2} x^{2}}\left[\vec{R}_{\perp}^{4}+2 m^{2} \vec{R}_{\perp}^{2}\left(1-8 x^{2}\right)+m^{4}\left(1-4 x^{2}\right)^{2}\right] .
\end{aligned}
$$

To calculate the traces (50), we need the following scalar products:

$$
k \cdot Q=2 m^{2}+(1-x)\left(s_{12}-4 m^{2}\right), \quad k \cdot p=k \cdot Q / 2, \quad p \cdot Q=Q^{2} / 2=2 m^{2} .
$$

Substituting (50) into (49), we find:

$$
\begin{aligned}
& A(\mu)=\frac{8 \pi \alpha}{(2 \pi)^{3}} \int d^{2} R_{\perp} \int_{0}^{1 / 2} \frac{\left[\vec{R}_{\perp}^{2}(1-2 x)+m^{2}\left(1+4 x^{2}\right)\right]}{\left[\vec{R}_{\perp}^{2}+m^{2}(1-2 x)^{2}\right]\left[\vec{R}_{\perp}^{2}+m^{2}(1-2 x)^{2}+2 \mu^{2} x\right]} d x, \\
& B(\mu)=-\frac{8 \pi \alpha}{(2 \pi)^{3}} \int d^{2} R_{\perp} \int_{0}^{1 / 2} \frac{\left[\vec{R}_{\perp}^{2}(1-4 x)+m^{2}(1-2 x)^{2}(1+2 x)\right]}{\left[\vec{R}_{\perp}^{2}+m^{2}(1-2 x)^{2}\right]\left[\vec{R}_{\perp}^{2}+m^{2}(1-2 x)^{2}+2 \mu^{2} x\right]} d x,
\end{aligned}
$$




$$
C(\mu)=\frac{4 \pi \alpha}{(2 \pi)^{3}} \int d^{2} R_{\perp} \int_{0}^{1 / 2} \frac{\left[\vec{R}_{\perp}^{4}+2 m^{2} \vec{R}_{\perp}^{2}\left(1-8 x^{2}\right)+m^{4}\left(1-4 x^{2}\right)^{2}\right]}{2 m^{2} x\left[\vec{R}_{\perp}^{2}+m^{2}(1-2 x)^{2}\right]\left[\vec{R}_{\perp}^{2}+m^{2}(1-2 x)^{2}+2 \mu^{2} x\right]} d x
$$

The integral (54) for $C$, which is the coefficient in front of the structure proportional to $\omega_{\rho}$, diverges quadratically at $R_{\perp} \rightarrow \infty$ and logarithmically at $x=0$. The integral (52) for $A$ logarithmically diverges at $R_{\perp} \rightarrow \infty$. The integral (53) for $B$ at $R_{\perp} \rightarrow \infty$ has the asymptotic expression:

$$
B(\mu) \propto \int_{0}^{\infty} \frac{d R_{\perp}}{R_{\perp}} \int_{0}^{1 / 2}(1-4 x) d x
$$

Since the integral over $x$ is zero, $B(\mu)$ is finite.

The regularization of $A(\mu)$ proceeds as follows. The integral over $x$ in $A(\mu)$ can be done analytically. In the Pauli-Villars regularization scheme, we should take the difference $A(\mu)-A(\Lambda)$ and calculate the convergent integral over $R_{\perp}$. Equivalently, but technically easier, we calculate $A(\mu, L)$ with the cutoff $L$ in the variable $R_{\perp}$, take the difference $A(\mu, L)-A(\Lambda, L)$ and then take the limit $L \rightarrow \infty$. The result is analytic, but lengthy. In the limits $\mu \rightarrow 0$ and $\Lambda \rightarrow \infty$ we obtain eq.(34), which coincides with the result calculated in the Feynman formalism [14].

\section{B Calculation of $a, b$ and $C_{1}^{\text {ren }}$}

According to eqs.(40) and (41), the self-energy $\Sigma_{R}(p)$ is determined by the scalar functions $a$ and $b$. As we will see below, $a$ and $b$ are determined by the coefficients $A_{1}\left(p^{2}\right)$ and $B_{1}\left(p^{2}\right)$ in the general decomposition (37) and by their combinations in the limit $p^{2} \rightarrow m^{2}$.

From (37) we find the coefficients $A_{1}\left(p^{2}\right)$ and $B_{1}\left(p^{2}\right)$ :

$$
A_{1}=\frac{1}{4} \operatorname{Tr}[\Sigma(p)], \quad B_{1}=\frac{m}{\omega \cdot p} \operatorname{Tr}[\Sigma(p) \hat{\omega}] .
$$

Substituting here eq.(42) for $\Sigma(p)$ we get:

$$
\begin{aligned}
& A_{1}\left(p^{2}\right)=\frac{\alpha m}{\pi^{2}} \int \frac{\pi d R_{\perp}^{2} d x}{R_{\perp}^{2}+(1-x) m^{2}+x\left[\mu^{2}+(1-x) p^{2}\right]} \\
& B_{1}\left(p^{2}\right)=-\frac{\alpha m}{2 \pi^{2}} \int \frac{\pi d R_{\perp}^{2} x d x}{R_{\perp}^{2}+(1-x) m^{2}+x\left[\mu^{2}+(1-x) p^{2}\right]} .
\end{aligned}
$$

These integrals diverge logarithmically.

Comparing (37) with (39) and taking into account (40), we find:

$$
A_{1}\left(p^{2}\right)+B_{1}\left(p^{2}\right) \frac{\hat{p}}{m}=A_{0}+(\hat{p}-m) B_{0}+(\hat{p}-m)^{2} \mathcal{M}(p) .
$$

From here we can express the constants $A_{0}$ and $B_{0}$ through $A_{1}$ and $B_{1}$ :

$$
\begin{aligned}
& A_{0}=\frac{1}{4 m} \operatorname{Tr}\left[\left(A_{1}\left(p^{2}\right)+B_{1}\left(p^{2}\right) \frac{\hat{p}}{m}\right)(\hat{p}+m)\right]_{p^{2}=m^{2}}=A_{1}\left(m^{2}\right)+B_{1}\left(m^{2}\right) \\
& B_{0}=\frac{1}{4 m\left(p^{2}-m^{2}\right)} \operatorname{Tr}\left[\left(A_{1}\left(p^{2}\right)+B_{1}\left(p^{2}\right) \frac{\hat{p}}{m}-A_{0}\right)(\hat{p}+m)^{2}\right]_{p^{2} \rightarrow m^{2}}
\end{aligned}
$$


We thus obtain:

$$
\begin{aligned}
& A_{0}=\frac{\alpha m}{2 \pi^{2}} \int \frac{(2-x) \pi d R_{\perp}^{2} d x}{R_{\perp}^{2}+(1-x)^{2} m^{2}+x \mu^{2}} \\
& B_{0}=-\frac{\alpha}{2 \pi^{2}} \int \frac{x\left[R_{\perp}^{2}-\left(3-4 x+x^{2}\right) m^{2}+x \mu^{2}\right] \pi d R_{\perp}^{2} d x}{\left[R_{\perp}^{2}+(1-x)^{2} m^{2}+x \mu^{2}\right]^{2}}
\end{aligned}
$$

These integrals also diverge logarithmically.

From (58), and taking into account eq.(41) for $\mathcal{M}(p)$, we get:

$$
(\hat{p}-m)^{2}(a+(\hat{p}+m) b)=A_{1}\left(p^{2}\right)+B_{1}\left(p^{2}\right) \frac{\hat{p}}{m}-A_{0}-(\hat{p}-m) B_{0} .
$$

This allows finding out $a$ and $b$ :

$$
\begin{aligned}
a & =\frac{1}{4 p^{2}\left(p^{2}-m^{2}\right)} \operatorname{Tr}\left[\left(A_{1}\left(p^{2}\right)+B_{1}\left(p^{2}\right) \frac{\hat{p}}{m}-A_{0}-(\hat{p}-m) B_{0}\right)(\hat{p}+m) \hat{p}\right] \\
& =\frac{A_{1}\left(p^{2}\right)+B_{1}\left(p^{2}\right)-A_{0}}{p^{2}-m^{2}} \\
b & =\frac{1}{4 p^{2}\left(p^{2}-m^{2}\right)^{2}} \operatorname{Tr}\left[\left(A_{1}\left(p^{2}\right)+B_{1}\left(p^{2}\right) \frac{\hat{p}}{m}-A_{0}-(\hat{p}-m) B_{0}\right)(\hat{p}+m)^{2} \hat{p}\right] \\
& =\frac{2 m\left(A_{1}\left(p^{2}\right)-A_{0}\right)}{\left(p^{2}-m^{2}\right)^{2}}+\frac{\left(p^{2}+m^{2}\right) B_{1}\left(p^{2}\right)}{m\left(p^{2}-m^{2}\right)^{2}}-\frac{B_{0}}{p^{2}-m^{2}} .
\end{aligned}
$$

Substituting here the above expressions for $A_{1}, B_{1}, A_{0}$ and $B_{0}$, we get:

$$
\begin{gathered}
a=\frac{\alpha m}{2 \pi} \int \frac{x\left(2-3 x+x^{2}\right) d R_{\perp}^{2} d x}{\left[R_{\perp}^{2}+m^{2}(1-x)^{2}\right]\left[R_{\perp}^{2}+m^{2}(1-x)(1-(1-\rho) x)\right]} . \\
b=-\frac{\alpha}{2 \pi} \int \frac{x^{2}(1-x)\left[R_{\perp}^{2}-m^{2}\left(3-4 x+x^{2}\right)\right] d R_{\perp}^{2} d x}{\left[R_{\perp}^{2}+m^{2}(1-x)^{2}+\mu^{2} x\right]^{2}\left[R_{\perp}^{2}+m^{2}(1-x)(1-(1-\rho) x)+\mu^{2} x\right]} .
\end{gathered}
$$

We omitted in $a$ the photon mass $\mu$, since that integral has no infrared divergence, and introduced the notation: $\rho=\left(m^{2}-p^{2}\right) / m^{2}$. Integrating over $R_{\perp}^{2}$ and $x$ and keeping in $b$ the leading term in $\log \left(\mu^{2} / m^{2}\right)$ only, we obtain eqs.(43).

One can similarly calculate the coefficient $C_{1}$ determining the $\omega$-dependent part of $\Sigma(p)$. It is given by:

$$
\begin{aligned}
C_{1}\left(p^{2}\right) & =\frac{1}{4 \omega \cdot p} \operatorname{Tr}\left[\Sigma(p)\left(\hat{p}-\frac{p^{2} \hat{\omega}}{\omega \cdot p}\right)\right] \\
& =-\frac{\alpha}{4 \pi^{2} \omega \cdot p} \int \frac{\left[2 R_{\perp}^{2}+m^{2}\left(2-3(1-\rho) x^{2}\right)\right] \pi d R_{\perp}^{2} d x}{\left[R_{\perp}^{2}+m^{2}(1-x)(1-(1-\rho) x)+\mu^{2} x\right] x}
\end{aligned}
$$

It is quadratically divergent in the variable $R_{\perp}$ and is logarithmically divergent at $x=0$, despite the finite photon mass $\mu$. Note that the standard Pauli-Villars regularization is not enough to make it finite.

We renormalize this scalar function in the standard way [11]:

$$
C_{1}^{r e n}\left(p^{2}\right)=C_{1}\left(p^{2}\right)-C_{1}\left(m^{2}\right)-\left.\left(p^{2}-m^{2}\right) \frac{C_{1}\left(p^{2}\right)}{d p^{2}}\right|_{p^{2}=m^{2}}
$$


After that it becomes finite:

$$
\begin{aligned}
C_{1}^{r e n}\left(p^{2}\right) & =-\frac{\alpha m^{4} \rho^{2}}{4 \pi^{2}(\omega \cdot p)} \int \frac{\left[R_{\perp}^{2}(2-5 x)+m^{2}\left(2-5 x+3 x^{2}\right)-3 \mu^{2} x^{2}\right] x(1-x) \pi d R_{\perp}^{2} d x}{\left[R_{\perp}^{2}+m^{2}(1-x)(1-(1-\rho) x)+\mu^{2} x\right]\left[R_{\perp}^{2}+m^{2}(1-x)^{2}+\mu^{2} x\right]^{2}} \\
& =-\frac{\alpha m^{2} \rho}{4 \pi(\omega \cdot p)}\left[1-\frac{2-\rho}{2(1-\rho)} \log \rho+\log \frac{\mu^{2}}{m^{2}}\right]
\end{aligned}
$$

\section{References}

[1] S. J. Brodsky, H.-C. Pauli, S. Pinsky, Phys. Reports 301 (1998) 299.

[2] J. Carbonell, B. Desplanques, V.A. Karmanov and J.-F. Mathiot, Phys. Reports, 300 (1998) 215.

[3] S.J. Brodsky, R. Roskies, R. Suaya, Phys. Rev. D8 (1973) 4574.

[4] D. Mustaki, S. Pinsky, J. Shigemitsu and K. Wilson, Phys. Rev. D43 (1991) 3411.

[5] D. Mustaki and S. Pinsky, Phys. Rev. D45 (1992) 3775.

[6] R.J. Perry and A. Harindranath, Phys. Rev. D43 (1991) 4051.

[7] N.E. Ligterink, B.L.G. Bakker, Phys. Rev. D52 (1995) 5917.

[8] K.G. Wilson, Phys. Rev. 140 (1965) B445, Phys. Rev. D2 (1970) 1438.

[9] S. Glazek and R.J. Perry, Phys. Rev. D45 (1992) 3740.

[10] V.A. Karmanov and J.-F. Mathiot, Nucl. Phys. A602 (1996) 388.

[11] C. Itzykson and J.-B. Zuber, Quantum Field Theory, McGraw-Hill book company, 1980 .

[12] F. Mandl and G. Shaw, Quantum Field Theory, John Wiley and Sons Ltd, 1984.

[13] E. Lifshits and L. Pitaevsky, Relativistic Quantum Theory, part 2, sect. 117, Pergamon Press, UK, 1974.

[14] R. Karplus and A. Klein, Phys. Rev. 87 (1952) 848; R. Barbieri et al., Phys. Lett. B57 (1975) 455; W. Celmaster, Phys. Rev. D19 (1979) 1517; W. Buchmüller, S.-H.H. Tye, Phys. Rev. D24 (1981) 132.

[15] F. Bissey, J.-J. Dugne and J.-F. Mathiot, Dynamical relativistic corrections to the leptonic decay width of heavy quarkonia, in preparation.

[16] V.A. Karmanov, J.-F. Mathiot and D. Bernard, Non-perturbative renormalization in a scalar model, in preparation. 